\title{
Caracterização e Gerenciamento de Ecossistemas Digitais de Inovação Social
}

\section{Luciana de Oliveira Vilanova Chueri, Rodrigo Pereira dos Santos}

\author{
Programa de Pós-graduação em Informática \\ Universidade Federal do Estado do Rio de Janeiro \\ Av. Pasteur, 458 - Rio de Janeiro - CEP: 22290-240 - Rio de Janeiro \\ \{luciana.chueri, rps\}@uniriotec.br
}

\begin{abstract}
Recent studies in social innovation ecosystems research area claim for new technological solutions for providing support to social innovation actors in order to foster collaboration, cocreation, and knowledge and competencies sharing inside these environments. Such support aims at improving the development of social innovation projects, the dissemination and generation of more effective social innovations, and the use of existing knowledge. This PhD research proposes an approach for exploring digital ecosystem concepts to build a solution to support a Social Innovation Digital Ecosystem where social innovation actors interact and collaborate through the support provided by a common technological platform.
\end{abstract}

Resumo. Estudos recentes na área de ecossistemas de inovação social expõem a necessidade de criação de soluções tecnológicas de suporte aos atores de inovação social para fomentar a colaboração, cocriação, compartilhamento de conhecimento e competências nestes ambientes. Este apoio visa facilitar a condução de projetos de inovação social, geração de inovações sociais mais efetivas e utilização de conhecimento existente. Esta pesquisa de doutorado propõe uma abordagem que explora conceitos relacionados a ecossistemas digitais visando construir uma solução de apoio a um Ecossistema Digital de Inovação Social no qual atores de inovação social interagem e colaboram com apoio de uma plataforma tecnológica.

\section{Introdução}

Atualmente, existe um crescente consenso entre a comunidade de pesquisa, praticantes e formuladores de políticas públicas de que as inovações tecnológicas têm enfrentado um dilema entre eficiência e desenvolvimento socioambiental. Após todo o avanço tecnológico vivenciado nas últimas décadas, seus resultados parecem ineficazes na resolução de desafios complexos, sejam eles sociais, econômicos, políticos e ambientais [Howaldt et al. 2016]. Neste cenário, cresce o reconhecimento das inovações sociais na resolução destas questões [Murray et al. 2010].

Inovações sociais são novas soluções (produtos, serviços etc.) que simultaneamente atendem a uma necessidade social de forma mais eficiente que soluções existentes, trazem novas ou melhoradas capacidades (e relacionamentos) e também viabilizam um melhor uso de recursos, melhorando a capacidade de atuação da sociedade [Caulier-Grice et al. 2012]. Estas inovações diferem da inovação tecnológica em termos do processo que elas demandam (cooperativo e colaborativo) e em termos de seu propósito (atender a necessidades sociais e criar novos relacionamentos e empoderamento 
dos participantes) [Murray et al. 2010; Howaldt et al. 2016]. Consequentemente, a gestão da inovação social se distingue da inovação tecnológica e a condução de processos nesta área requer metodologias, modelos e processos distintos dos tradicionalmente utilizados [Silva e Bignetti 2012].

Atualmente, existe numerosas e diversas iniciativas de inovação social sendo desenvolvidas de forma isolada, onde cada iniciativa trilha seu próprio caminho e cada gestor faz sua própria interpretação de conceitos e processos. Neste caminho isolado, uma iniciativa pode até resultar em uma inovação social, mas de forma mais difícil e sem conexão ou colaboração com outros atores. Neste contexto, surge a necessidade dos atores em desenvolver redes e aumentar a participação de atores intermediários, de forma a aumentar o desenvolvimento e a disseminação de inovações sociais [Howaldt et al. 2016; Domanski e Kaletka 2018].

\section{Apresentação do Problema}

Algumas necessidades reportadas em ambientes onde atores desenvolvem inovações sociais são: i) combinar diferentes conhecimentos, recursos e competências; ii) viabilizar acesso a inovações existentes; iii) prover estrutura e suporte para realizar desenvolvimento e escalabilidade de inovações sociais; iv) prover mecanismos, processos e sistemas para os atores provenientes de diversos setores da economia (organizações, governos, universidades etc.); v) prover mecanismos que viabilizem a aplicação de inovações sociais em diferentes contextos; e vi) tratar a complexidade dos processos de inovação social [Gabison e Pesole 2014; Sgaragli 2014]. Um mapeamento sistemático realizado sobre o assunto indicou que os maiores desafios se referem aos atores (falta de habilidades, competências para desenvolver os projetos, falta de comprometimento e falta de engajamento), aos processos (gerenciamento do projeto, mudanças, processos de design, avaliação e técnicas de apoio) e a raras soluções tecnológicas de apoio a estes ambientes [Chueri e Araujo 2018].

Recentemente, observou-se a utilização da metáfora de ecossistemas para estes ambientes, porém com poucas definições de ecossistemas de inovação social e estudos encontrados na literatura [Sgaragli 2014; Howaldt et al. 2016; Braithwaite 2018; Domanski e Kaletka 2018]. Para Braithwaite (2018), um ecossistema de inovação social descreve um ambiente de suporte com inovações múltiplas e interconectadas que precisa ser criado para viabilizar que inovações sociais atinjam o estágio de mudança sistêmica. Segundo Manzini (2015), estes ecossistemas são cruciais para: i) promover, apoiar e desenvolver iniciativas bem-sucedidas de inovação social; ii) criar as redes que permitem aos atores se envolver; e iii) compartilhar ideias e inovação. Desta forma, é importante criar uma infraestrutura de apoio representada por uma plataforma bem estruturada para dar apoio e conectar as diferentes iniciativas.

Além disso, um estudo exploratório em um ecossistema real de inovações sociais realizado indicou a importância do ambiente em que as inovações sociais são desenvolvidas [CHUERI 2018]. Além disso, foi percebido que este ambiente possuía as seguintes características: i) atores de diferentes setores da economia e dispersos geograficamente; ii) atores externos transitando no ambiente; iii) atores fazendo uso de comunicação síncrona e assíncrona; iv) atores colaborando em prol de um objetivo comum, isto é, desenvolvimento de inovações sociais; v) atores com necessidade de atuar 
colaborativamente, intra e inter-projetos; vi) ausência de fronteira organizacional; e vii) ausência de regulação ou controle formal sobre os atores.

As percepções discutidas, resultantes do mapeamento sistemático, do estudo exploratório e das definições de ecossistemas de inovações sociais, expõem a necessidade de criação de soluções tecnológicas de suporte aos atores destes ecossistemas. Elas podem fomentar a colaboração, cocriação, troca e compartilhamento de ideias, informações e conhecimento, obter o apoio necessário para a condução de projetos de inovação social e gerar inovações sociais mais efetivas e que atendam a mais pessoas. Considerando a necessidade de suporte tecnológico, foram pesquisadas categorias de ecossistemas que fizessem uso de tecnologias da informação e da comunicação (TICs). Por suas características serem consideradas aplicáveis a um ecossistema de inovação social, decidiu-se utilizar um ecossistema digital (do inglês, digital ecosystem) ou ECODig.

Um ECODig consiste de uma infraestrutura digital auto-organizável com o intuito de criar um ambiente digital para as organizações conectadas em rede, provendo apoio à cooperação, compartilhamento de conhecimento e desenvolvimento de tecnologias adaptativas e abertas [IEEE 2007; Magdaleno e Araujo 2015]. A utilização de elementos e características de um ecossistema digital em uma nova categoria de ecossistemas para apoio a atores de inovações sociais leva à seguinte questão de pesquisa (QP): "Que características e componentes contribuem para o gerenciamento de um ecossistema digital de inovação social?”.

\section{Proposta de Solução}

Esta pesquisa propõe a criação de um Ecossistema Digital de Inovação Social (ECODIS), utilizando as características inerentes a um ecossistema digital (ECODig) como base para criação de uma nova categoria de ecossistema para atender aos atores de ecossistemas de inovação social. Um ECODIS é um ecossistema que proporciona a interação de uma comunidade de atores formada por organizações (sociais ou orientadas a lucro), universidades, empreendedores, indivíduos e governo, com o objetivo de gerar inovações sociais (produtos, processos e serviços) para atender aos desafios da sociedade, por meio de uma plataforma tecnológica (de suporte aos atores, seus relacionamentos e artefatos) e de um processo colaborativo, inclusivo e aberto.

A partir das percepções obtidas com a realização do mapeamento, do estudo exploratório e das necessidades de ecossistemas de inovações sociais [Sgaragli 2014; Manzini 2015; Braithwaite 2018; Domanski e Kaletka 2018], foi elaborado um conjunto preliminar de requisitos (REQ) de um ECODIS, listado a seguir:

REQ1: Um ECODIS deve ser definido de maneira simples a partir de um conjunto mínimo de conceitos e suas relações;

REQ2: Um ECODIS deve conter uma base de conhecimento composta por um glossário de termos de ECODIS (conceitos e respectivos significados) e toda a documentação referente aos atores e seus relacionamentos;

REQ3: Um ECODIS deve conter um repositório para armazenamento dos artefatos (software, modelos, documentos) gerados no desenvolvimento de uma inovação social; 
REQ4: Um ECODIS deve conter uma ferramenta de suporte à gestão do conhecimento para a busca de informações da base do conhecimento o arquivamento, busca e recuperação de dados;

REQ5: Um ECODIS deve possuir uma ferramenta para viabilizar a colaboração, cocriação e compartilhamento de ideias e inovação entre os atores;

REQ6: Um ECODIS deve conter uma ferramenta de suporte à gestão de competências do ecossistema para identificar lacunas de competência dos projetos e aumentar a interação entre os atores do ecossistema;

REQ7: Um ECODIS deve conter uma ferramenta de apoio ao gestor do ecossistema visando coordenar estratégias de sobrevivência e agregação dos atores em torno de uma plataforma.

A proposta de solução da pesquisa foi definida e aprovada em uma defesa de exame de qualificação. Os próximos passos da pesquisa estão divididos em três etapas, onde cada etapa é alimentada pelos resultados de avaliação da anterior:

1. definição dos elementos para a caracterização do ECODIS: a) Glossário de termos; b) Modelo conceitual; e c) Conjunto de elementos (definição de atores, papéis, ciclo de vida, relacionamentos, entre outros) e suas relações nos ECODIS com seus respectivos modelos. Esta definição de elementos possui como insumos um modelo de ECODig (resultante de um mapeamento sistemático sobre o tema, que está em andamento) e uma pesquisa em ferramentas de apoio a inovações sociais (também em andamento);

2. definição de um conjunto de requisitos que definam como deve ser o suporte a ambientes de inovação social com características de ECODig, gerando um conjunto de requisitos (funcionais e não funcionais) da plataforma do ECODIS, especificação da plataforma de apoio ao gerenciamento do ecossistema e ajustes nos elementos avaliados na etapa anterior;

3. elaboração do projeto, implementação e testes de aceitação da plataforma tecnológica do ECODIS.

Com relação ao enfoque de solução, foram identificados alguns trabalhos relacionados: i) o Observatório de Inovação Social de Florianópolis [OBISF 2019] oferece uma plataforma online que apresenta a cartografia do ecossistema de inovação social da cidade, permitindo a visualização de iniciativas de inovação social e organizações de apoio à inovação social, além de gráficos apresentando a interação entre atores e gráficos por localização e tipo de iniciativa; e ii) Gomes (2017) propõe uma arquitetura incluindo elementos de ecossistemas de inovação e de software para auxiliar a publicação de dados em ecossistemas de inovação com tecnologias semânticas.

O diferencial da proposta apresentada nesta pesquisa em relação aos trabalhos relacionados é fornecer uma solução tecnológica que apoie o ator que idealiza e desenvolve uma inovação social, nas interações e na colaboração com os outros atores do ecossistema. A proposta de solução utilizará os elementos discutidos em Santos et al. (2016) como referencial teórico para as atividades de caracterização do ecossistema e a pesquisa de Santos (2016) como referencial para as etapas de concepção e gerenciamento de um ecossistema. 


\section{Projeto de Avaliação da Solução}

A avaliação da solução é dividida em três etapas, a saber: i) avaliação dos elementos para caracterização do ECODIS por meio de uma pesquisa de opinião (survey); ii) avaliação dos requisitos e da especificação da plataforma de apoio ao ecossistema, que será realizada junto a especialistas em inovações sociais e em ecossistemas por meio de um estudo de grupo focal (focus group); e iii) avaliação da plataforma tecnológica do ECODIS por meio de um estudo de caso para verificar aspectos de facilidade de uso e utilidade em cada estágio de desenvolvimento de uma inovação social. Ao final de cada etapa, os resultados da pesquisa serão publicados e utilizados para a realização de ajustes nos elementos criados nas etapas anteriores. No final da terceira etapa, será publicada a solução, contendo as limitações do trabalho, ameaças à validade e questões éticas.

\section{Atividades Realizadas}

O início da pesquisa foi dedicado à investigação do campo de pesquisa de inovações sociais, o que resultou na elaboração do capítulo sobre este assunto [Chueri 2017] em um livro na área de inovação. Em seguida, foram investigados problemas relacionados ao desenvolvimento de projetos de inovações sociais, sendo inclusive realizado e publicado um mapeamento sistemático de literatura com este propósito [Chueri e Araujo 2018]. Os desafios resultantes do mapeamento serviram de base para condução de um estudo exploratório em um ecossistema real de inovações sociais. Um dos autores atuou no ecossistema na colaboração do planejamento de atividades de mentoria no ecossistema e, como resultado, foi publicado um artigo sobre este ecossistema de inovações sociais [Nunes et al. 2018].

\section{Considerações Finais}

A proposta desta pesquisa é investigar, propor e avaliar um conjunto de elementos para caracterização de um ECODIS e uma plataforma digital de apoio aos atores do ecossistema. Pretende-se fornecer contribuições científicas a pesquisadores que atuam com ecossistemas de inovação social e a pesquisadores de sistemas de informação que investigam ecossistemas digitais, pois estudos envolvendo estes dois ecossistemas são carentes na literatura. As contribuições se concretizarão nas publicações a serem divulgadas ao final de cada etapa de avaliação da pesquisa. Em termos de artefatos computacionais, espera-se que a plataforma promova um apoio real e relevante a pesquisadores de sistemas de informação que trabalham com ecossistemas digitais, atores participantes de ecossistemas de inovações sociais e profissionais da indústria de software interessados em soluções na área de ecossistemas digitais.

\section{Agradecimentos}

O presente trabalho foi realizado com apoio da Coordenação de Aperfeiçoamento de Pessoal de Nível Superior - Brasil (CAPES) - Código de Financiamento 001.

\section{Referências}

Braithwaite, P. (2018) "Building Change Trust: Social Innovation Ecosystems", Disponível em: $<$ https://www.buildingchangetrust.org/download/files/BCT_SIE_Report_Final.pdf.>

Caulier-Grice, J., Davies, A., Patrick, R., \& Norman, W. (2012). Defining social innovation. TEPSIE, European Commission - 7th Framework Programme, Brussels: European Commission, DG Research. 
Chueri, L.O.V. (2017) "Inovação Social”. In: Araujo, R.M., Chueri, L.O.V. (eds.), Pesquisa e Inovação: Visões e Interseções, Rio de Janeiro: PUBL!T Soluções Editoriais, p. 266-281.

Chueri, L.O.V. (2018) "Gerenciamento e monitoramento de Ecossistemas Digitais de Inovação Social”. Exame de Qualificação ao Doutorado em Informática. Programa de Pós-Graduação em Informática Universidade Federal do Estado do Rio de Janeiro, Rio de Janeiro, Brasil, 166p.

Chueri, L.O.V. e Araujo, R. M. (2018) "How social innovation projects are managed? Answers from a literature review”, European Public \& Social Innovation Review, v. 3, p. 23-36.

Domanski, D. e Kaletka, C. (2018) "Social Innovation Ecosystems", in: Title: Atlas of Social Innovation, New Practices for a Better Future, Editors: Howaldt, Jürgen / Kaletka, Christoph / Schröder, Antonius / Zirngiebl, Marthe, Editora: Sozialforschungsstelle, TU Dortmund University: Dortmund.

Gabison, G. e Pesole, A. (2014) “An Overview of Models of Distributed Innovation - Open Innovation, User Innovation and Social Innovation”, ISSN 1831-9424, doi:10.2791/347145. Luxembourg: Publications Office of the European Union.

Gomes, M. S. (2017) "Proposta de Arquitetura para Ecossistema de Inovação em Dados Abertos", Dissertação (mestrado) - Universidade Federal de Santa Catarina, Centro Tecnológico, Programa de Pós-Graduação em Engenharia e Gestão do Conhecimento, Florianópolis, SC, 104 p.

Howaldt, J., Kaletka, C., Schröder, A., Terstriep, J., Christoph, R., Dieter, T. (2016): “Comparative Analysis (Mapping 1) - Mapping the World of Social Innovation: A Global Comparative Analysis across Sectors and World Regions (D1.4)", Disponível em: <https://www.si-drive.eu/wpcontent/uploads/2016/07/SI-DRIVE-D1-4-Comparative-Analysis-2016-08-15-final.pdf.>

IEEE (2007) "Inaugural IEEE International Digital Ecosystems and Technologies Conference", http://www.ieeedest.curtin.edu.au/2007/ index.php.

Manzini, E. (2015) "Design, When Everybody Designs: An Introduction to Design for Social Innovation", Cambridge, Massachusetts: The MIT Press.

Murray, R., Caulier-Grice, J.; Mulgan, G. (2010) “The Open Book of Social Innovation”, London, NESTA, The Young Foundation, Disponível em: <http://www.nesta.org.uk/publications/open-book-socialinnovation>

Nunes, L.P., Chueri, L.O.V., Alencar, M.S.M. (2018) "Una experiencia de apoyo a proyectos de innovación social en una universidad pública brasileña: NICS/UNIRIO”, VI Simposio Internacional de Innovación Social y Tecnológico, 27 a 28 de Setembro, Medellín, Colombia.

OBISF. (2019) “O que é o Observatório de Inovação Social”, Observatório de Inovação Social de Florianópolis, Disponível em: <http://www.observafloripa.com.br/is-page//methodology.>

Santos, R.P. (2016) "Managing and Monitoring Software Ecosystem to Support Demand and Solution Analysis", Tese (doutorado) - UFRJ/ COPPE/ Programa de Engenharia de Sistemas e Computação.

Santos, R. P., Viana, D., Maciel, C. (2016) "Ecossistemas de Software: Uma Visão sobre Fatores Técnicos, Humanos e Organizacionais", In I. Gasparini, M. Mota (Org.), Livro dos Tutoriais do XV IHC, 15ed. Porto Alegre: SBC, v. C, pp. 70-90.

Sgaragli, F. (2014) "Enabling Social Innovation Ecosystems for Community-led Territorial Development", Fondazione Giacomo Brodolini, Rome.

Silva, S. B. e Bignetti, L. P. (2012) “A inovação social e a Dinâmica de Inovação Aberta na Rede Brasileira de Living Labs”, Encontro da ANPAD, http://www.anpad.org.br/admin/pdf/2012_GCT1794.pdf. 